\title{
A SELF-ADJUSTING WEIGHTED MEDIAN FILTER FOR REMOVING IMPULSE NOISE IN IMAGES
}

\author{
Chun-Te Chen and Liang-Gee Chen
}

\author{
Department of Electrical Engineering, National Taiwan University, Taipei, Taiwan, R.O.C. \\ e-mail : d80049@video.ee.ntu.edu.tw lgchen@video.ee.ntu.edu.tw
}

\begin{abstract}
In this paper, an intelligent self-adjusting weighted median filter for removing impulsive noise in images is presented. Three main techniques are developed to implement this self-adjusting weighted median filter : an intelligent classifcation to divide the image data into the "corrupted" pixels and the "uncorrupted" pixels, an efficient algorithm to find the median output of any weight set, and a realistic training procedure without the noise free image to obtain the proper weights. Our simulations on some test images demonstrate that the proposed filter has less smoothing effect and smaller MAE or MSE measurement than the standard median filter. At the same time, the quality of filter output has enhanced significantly. Finally, a demonstration chip of this weighted median filter for the $5 \times 5$ window size is also presented in this paper.
\end{abstract}

\section{INTRODUCTION}

The Weighted Order Statistic Filter(WOSF), which is an extension of the Standard Median (SM) filter gives more weight to some values within the window so as to include both the spatial and rank information. The Weighted Median Filter(WMF) is a special example of the Weighted Order Statistic (WOS) filter. The WMF was first introduced by Justusson[1], and further discussed by Brownrigg[2] and others $[3,5,7,9,10]$. With a proper weights set, the WMF has an efficient impulsive noise suppression and an excellent image detail-preserving capability. It has been successfully applied in various areas such as noise reduction[4], image restoration, field interpolation, and image DPCM coding. Many theories about WMFs are being developed and focus on their deterministic and statistical properties[5].

There are two main items in the implementation of WMF: an algorithm to find the proper weight, and an efficient algorithm to find its output. The first attempt to implement the WM filter was made by Yl. Harji et. al[5] based on the Positive Boolean Function(PBF) and threshold decomposition. Then, the weighted order statistic filters based on the back-propagation algorithm[7] and the perception algorithm[9] have been proposed to obtain the optimal weight set. They adjust the weights to minimize the mean square error (MSE) or mean absolute error (MAE) between the filter's output and the desired noise free image. The existing designs, however, require a large memory and heavy computation during the filter training processing and during the actual filtering processing. These existing designs are too complex to implement on VLSI. In addition, the convergence rate is very slow. Another drawback is that these approaches always assume the desired signal is known during the training procedure.

There are three architectures[12] for the weighted order statistic filters : 1)stack-based WOS filter, 2)rank-order state machine architecture, and 3)sorting network architecture. The stack-based WOS filter requires $\mathrm{N}$ comparators as threshold level prior to the PBF implementation. Generally, a filter of $5 \mathrm{X} 5$ window size requires about $32 \mathrm{M}$ bytes of ROM to implement the PBF function[11]. In addition, it needs at least 8 times of memory access to generate 8 bits output. The processing speed of such a system is rather limited. Although the adder-tree[12] is alternative method to replace the PBF implementation, it is unefficient design because of $\mathrm{N}$ comparators. Although the rank-order state machine architecture can find the rank of each samples, it requires a long cyel time to extract the WMF's output. On the other hand, the bandwidth of the sorting networks are extended to contained the input samples and the corresponding weights. Recently, an alternative efficient method without threshold circuits for the weighted median filter is proposed in $[15,14]$.

In this paper, we propose an intelligent self-adjusting weighted median filter suitable for VLSI implementation. The weight of the filters is obtained by using the noise image. Then, the final output of the proposed filter is obtained by combining the "central" pixels and the weighted median filter's output at the filtering operation. Based on our simulations, the proposed filter has less smoothing effect and smaller MAE or MSE measurement than the standard median filter. At the same time, the quality of the filter output has enhanced significantly. We also construct a WMF demo chip with 5 X 5 window size in this paper.

\section{THE ALGORITHM OF A SELF-ADJUSTING WMF}

In this section, we propose a system block of the intelligent Self-Adjusting Weighted Median Filter (SAWMF) as shown in Fig. 1. We propose three main techniques to solve the optimal filtering problem : an intelligent classification to divide the image data into the "corrupted" pixels and the "uncorrupted" pixels, an efficient algorithm to find the median output of any weight set, and a realistic training procedure to obtain the proper weights. The final output is obtained by combining the "uncorrupted" pixels and the 


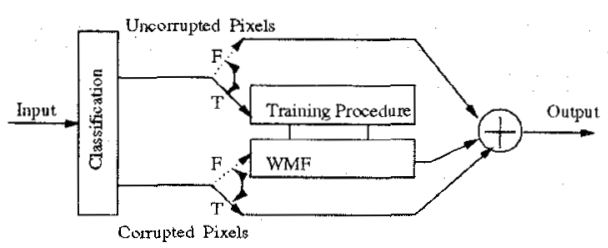

Figure 1: The system block of the self-adjusting weighted median filter.

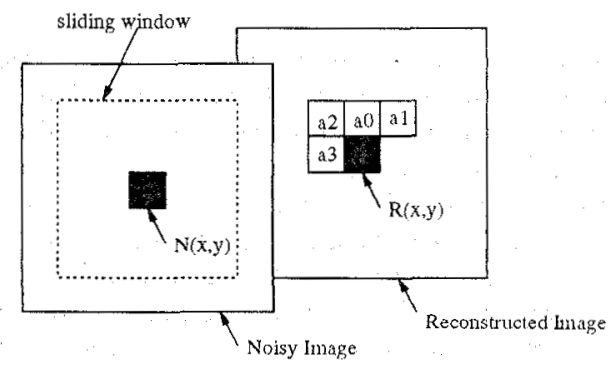

Figure 2: An intelligent classification with four neighboring pixels.

weighted median filter's output of the "corrupted" ones.

\subsection{An Intelligent Classification}

We propose a simple classification based on the correlation of the image data in order to divide the central pixels into "corrupted" and "uncorrupted" ones. Fig. 2 shows the central pixel and four neighboring pixels used to extract the spatial information. In the reconstructed image, the gray value of "a0", "a1","a2", and "a3" will have only a slight difference with that of $N(x, y)$. Hence, this spatial information can be used directly to classify the central pixels into the "uncorrupted" or "corrupted" ones. A similarity function is derived to extract the spatial information. The formula is :

$$
\mathrm{SF}=\mathrm{f}(|g(N(x, y))-g(a 0)|)+\mathrm{f}(|g(N(x, y))-g(a 1)|)
$$$$
+\mathrm{f}(|g(N(x, y))-g(a 2)|)+\mathrm{f}(|g(N(x, y))-g(a 3)|)
$$$$
\text { if }|g(N(\boldsymbol{x}, y))-g(a .)| \leq V_{t h} \text { then } f(.)=1 \text {, otherwise } 0 \text {, }
$$
where $\mathrm{g}($.$) means the gray value.$

The threshold value, $V_{t h}$, is used to control the tolerance of the similarity function. Based on the result of the similarity function, the central pixels can be exactly classified into the "corrupted" or "uncorrupted" ones. Since the correlation of the image data has much the same, the threshold value $V_{t h}$ can be directly assigned as 40 without a histogram evaluation in our experiment. It should be noted that this classification uses only the noisy image to extract the spatial information.

\subsection{A Hardware-Oriented Algorithm}

A hardware-oriented algorithm has been proposed in our previous works[14], which has a fixed input and constant iterations independent of the weight value defined.

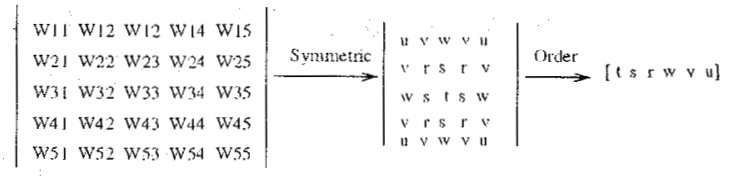

Figure 3: The parameters of the filter definition before being applied to the training algorithm.

\subsection{The SAWMF Training Procedure}

In the self-adjusting weighted median filter, the weight should be adjustable depending on the characteristics of the input data. To reduce the computation during the training procedure, a class of weighted median filters whose weights are symmetric about the window center is discussed. The number of the parameter to be adjusted is reduced to almost $1 / 4$ of the unsymmetric weighted median filters. The parameter of the filter design is given in Fig. 3. Since the desired signal is directly obtained from the natural noisy image, the previous training algorithm in $[5,9]$ can not be used to find the optimal solution. Let's assume that the. cost function (MAS or MSE) is convex and has a unique minimum value with multiple parameters. The initial value of the weighted median filter is set to 1 like that of standard median filter. Thus, the performance of this filter will be superior to the standard median filter. This training algorithm is summarized in the following:

Step 1: Cut a filtering window with an $n^{*} n$ size and its central pixel is $N(x, y)$.

Step 2: Obtain "a0", "a1", "a2", and "a3" from the reconstructed frame. Evaluate the similarity function, SF.

Step 3: IF SF $\geq 3$ then GOTO Step 4, otherwise GOTO Step 1 .

Step 4: In this case, the central pixel of the filtering window is uncorrupted. We adjust the weights $r, s, w, v$, and $u$ in sequence to minimize the MSE. That is because the filter output must be very close to the central pixels to reduce the smearing effect.

Step 5: If there is no further improvement, then EXIT, otherwise GOTO Step 1.

It should be noted that the central weight is always set to 1 without any adjustment in the training procedure. Although this procedure does not give us the exact weight set, it is easy to implement with VLSI technology.

\section{EXPERIMENTAL RESULTS AND COMPARISONS}

The Lena image consists of 512 X 512 pixels with 8 bit$s$ of resolution. The impulsive noise may have positive and negative impulse value 100 or -100 , respectively. The proposed SAWMF, the standard median filter(MF), CWM filter[4], RCRS filter[16], the previous Weighted Order Statistic Filters[7], and the WMF are all applied to these test images. The criteria of the Signal-to-Noise Ration (S$\mathrm{NR}$ ) is evaluated. The performance improvement of these filters over the standard median filter are summarized in Table 1. Since the noise model and the image data are different, this table only shows that the proposed design can perform very well in respect to the previous designs. 
Table 1: Summary of the performance improvement over the standard median filter for the proposed SAWMF, CWMF, RCRSF, WMF, and WOS. The window size is $5 \times 5$.

\begin{tabular}{|c|c|c|c|c|}
\hline Prob. & CWM & RCRS & WMF & SAWMF \\
\hline $5 \%$ & 4.66 & 1.88 & $\overline{6.38}$ & $\overline{6.18}$ \\
\hline $25 \%$ & 1.41 & 1.16 & 2.36 & 2.30 \\
\hline \multicolumn{5}{|c|}{ For the other WOS filters [7] } \\
\hline Prob. & $\begin{array}{l}\text { LMA } \\
\text { WOS }\end{array}$ & $\begin{array}{l}\text { LMS } \\
\text { WOS }\end{array}$ & Nonadaptive & Adaptive \\
\hline & 3.17 & 3.97 & 1.6 & 2.0 \\
\hline
\end{tabular}

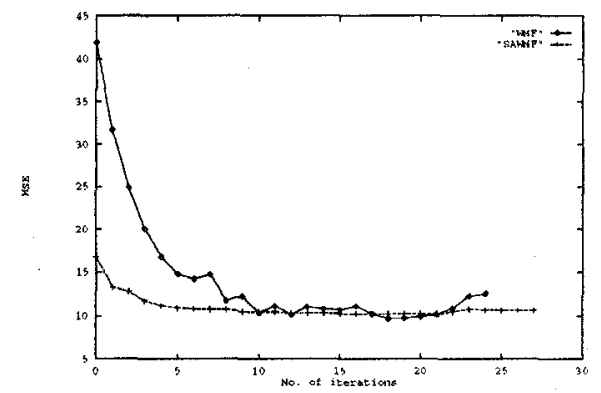

Figure 4: The convergence curve of the proposed SAWMF algorithm and the WMF algorithm under the MSE criterion.

The proposed SAWMF design has enhanced significantly in SNR. The convergence curve of the proposed SAWMF algorithm and the WMF algorithm under the MSE criterion is shown in Fig. 4. It needs about 25 iterations to find the proper weights. Fig. 5(a) shows a clear Lena image. Fig. 5 (b) shows the Lena image corrupted by $10 \%$ of impulsive noise. Fig. 5(c) and 5(d) present the images reconstructed by the standard median filter and the proposed SAWMF respectively for subjective evaluations. These object observation results agree with the SNR measurements as given in Table. 1.
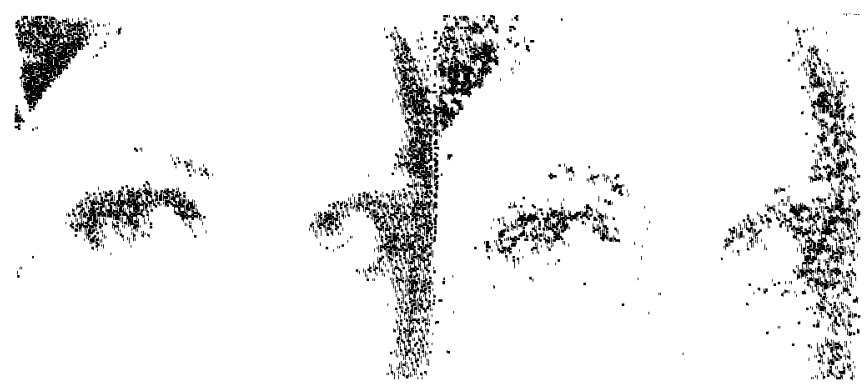

Figure 5: a) The original Lena image. b) The noisy image. c) The median filter's output. d) The proposed SAWMF output.

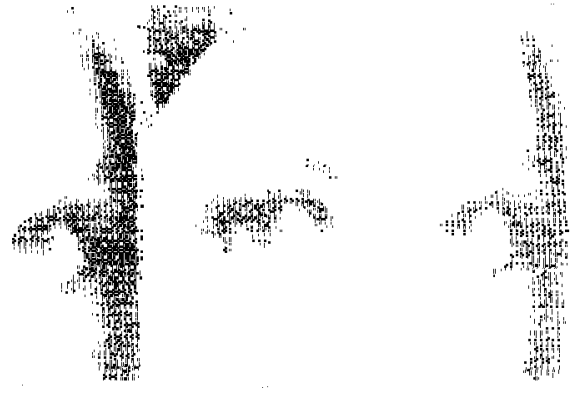

Table 2: Evaluation of proposed design and the stack-based PBF implementation $[8,12]$.

\begin{tabular}{|l||l||l|}
\hline Algorithm & $\begin{array}{l}\text { Stack-based } \\
\text { WOS }\{8,12\}\end{array}$ & $\begin{array}{l}\text { Our } \\
\text { algorithm }\end{array}$ \\
\hline Filter Design & WOS & WMF \\
\hline $\begin{array}{l}\text { Threshold cir- } \\
\text { cuits }\end{array}$ & 25 comparators & 0 \\
\hline $\begin{array}{l}\text { No. of PBF } \\
\text { No. of Adders }\end{array}$ & $\begin{array}{l}1 \text { PBF(25 adders }) \\
0\end{array}$ & $\begin{array}{l}0 \\
24 \text { adders }\end{array}$ \\
\hline \hline Area & $32 \mathrm{M}$ Bit ROM & $11.67 \mathrm{~mm}^{2}$ \\
\hline $\begin{array}{l}\text { Pipeline Cy- } \\
\text { cle(5*5) }\end{array}$ & larger than 20 ns & $6 \mathrm{~ns}(3.5 \mathrm{~V})$ \\
\hline
\end{tabular}

\section{VLSI OF SELF-ADJUSTING WEIGHTED MEDIAN FILTER}

The layout of the chip is displayed in Fig. 6 for the $5 \mathrm{X} 5$. window size. And the sum of the weights is limited to 255 . It takes 36 pins and about 2,500 standard cells. The active area of the weighted median finding is about $4.3 \times 3.7 \mathrm{~mm}^{2}$. The function of this chip is verified by Verilog Simulator. And the latency of the critical path or pipeline cycle is about $6 \mathrm{~ns}$ for $0.8 \mu \mathrm{m}$ standard cell library. The proposed chip can support weighted median filters, standard median filters, and order statistic filters, since the weights can down-loaded from the outside of the chip.

Table 2 summarizes the hardware resources for the stackbased WOS filter $[8,12]$ and the proposed architectures. Although the PBF function can be replaced by an adder tree[12], the previous designs require $N$ comparators as the threshold circuits. In addition, the cycle time is very long. However, an adaptive WOS filter algorithm[6] without threshold decomposition has been developed. It requires the use of a sequence operation to find the WOS filter output. In contract, the proposed design can directly evaluate the weighted median output without threshold circuit and pipeline in. bit-level with task interleaving processing. Therefore, the proposed architecture is very efficient in area and improves the throughput rate.

\section{CONCLUSIONS}

An original VLSI design of the Self-Adjusting Weighted Median Filter is presented in this paper. The main innovations are: an intelligent classification, an efficient algorithm to 


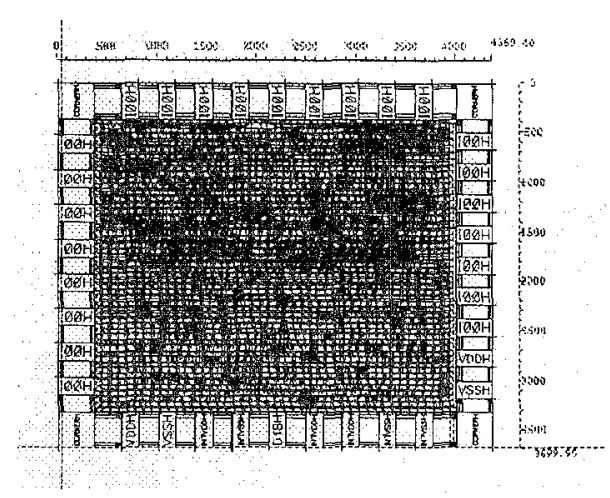

Figure 6: The final layout of the weighted median filter.

find the median output of any weight set, and a realistic training procedure to obtain the proper weights' value. At the training procedure, the characteristics of the image data are analyzed and used to "train" the Weighted Median Filter. Based on the intelligent classification, the "uncorrupted" pixels are extracted and used to adjust the weights in the filters. The final output is obtained by combining the "uncorrupted" pixels and those of the weighted median filter output. Our experiments on some test images demonstrate that the proposed filter has less smoothing effect and smaller MAE or MSE measurement than the standard median filter has. At the same time, the quality of the filter output has enhanced significantly. Finally, we suggest an efficient VLSI implementation of this weighted median filter, whose weights are adjustable. A demonstrate chip of this weighted median filter for the $5 \mathrm{X} 5$ window size is also presented in this paper. Some experimental results, complexity analysis, and VLSI system design are provided to demonstrate the advantages of the proposed SAWMF.

\section{REFERENCES}

[1] B. I. Justusson, " Median filtering: Statistic properties," in Topic in Applied Physics, Two-Dimensional Digital Signal Processing II, T. S. Huang, Ed. Berlin: Springer, 1981.

[2] D. R. K. Brownrigg, " The weighted median filter," Commun. Assoc. Comput. Mach., vol. 27, pp. 807818, Aug. 1984.

[3] P. D. Wendt, E. J. Coyle, and N. C. Gallagher, "Stack Filters," IEEE Trans. on Acoust., Speech, and Signal Processing, vol. 34, pp. 898-911, 1986.

[4] S. J. Ko and Y. H. Lee, "Center Weighted Median Filters and Their Application to Image Enhancement," IEEE Trans, on Circuits and Systems, vol. $38 \mathrm{pp}$. 984-993,1991

[5] O. Yli-Harja, J. Astola, and Y. Neuvo, "Analysis of the properties of median and weighted median filters using threshold logic and stack filter representation." IEEE Trans. on Acoust., Speech, and Signal Pracessing, vol. 39, No. 2, pp. 395-410, Feb. 1991.
[6] L. Yin and Y. A. Neuvo, 'An Adaptive WOS Filters Algorithm without Threshold Decomposition', Proceedings of ICASSP 93, pp. 930-933.

[7] L. Yin, J. T. Astola, and Y. A. Neuvo, 'Adaptive Multistage Weighted Order Statistic Filters Based on the Backpropogation Algorithm', IEEE Trans. on Signal Processing, vol. 42, pp. 419-423, 1993.

[8] L. Yin and Y. A. Neuvo, 'Fast Adaptive and Performance Characteristic of FIR-WOS hybrid Filter', IEEE Trans. on Signal Processing, vol. 42, pp. 16101628,1994

[9] Byeongiang Jeong and Yong Hoon Lee, 'Design of Weighted Order Statistic Filter Using the Perception Algorithm,', IEEE Trans. on Signal Processing, vol. 42, pp. 3264-3268, 1993.

[10] M. Gabbouj, "Weighted Median Filter-Striking Analogies to FIR Filters," in Circuits and Systems, Tutorials, Chris Toumazou, 1994 IEEE International Symposium on Circuits and Systems, London, pp. 521, May 1994.

[11] K. Chen, "Bit-serial Realizations of a Class on Nonlinear Filter Based on Positive Boolean Function," IEEE Trans. on Circuits and Systems, vol. 36, pp. 785794,1989 .

[12] G. B. Adams III, E. J. Coyle, L. Lin, L. E. Lucke, and K. K. Parhi, "Input Compression and Efficient VLSI Architectures for Rank Order Filter", Signal Processing, vol. 38 , No. 3 pp. 441-453, 1994.

[13] B. K. Kar and D. K. Pradhan, "A New Algorithm for Order Statistics and Sorting," IEEE Trans. on Signal Processing, vol. 41. No. 8, pp. 2688-2694, Aug. 1993.

[14] Chun-Te Chen, Liang-Gee Chen, and Jue-Hsuan Hsiao, "A Hardware-oriented Design for Weighted Median Filters," ASP-DAC'95 /CHDL'95/VLSI'95, Tokyo, JAPAN, pp. $441-445,1995$

[15] C. T. Chen, L. G. Chen, T. D. Chiueh, and J. H. Hsiao," Design and VLSI Implementation of Real-Time Weighted Median Filter," 1994 IEEE Asia-Pacific Conference on Circuits and Systems," Taipei, pp. 9197,1994

[16] R. C. Hardie and K. E. Barner, 'Rank Conditioned Rank Selection Filters for Signal Restoration,' IEEE Trans. on Image Processing, Vol. 3, pp.192- 206, Mar. 1994. 